\title{
The revised Beliefs About Voices Questionnaire
} (BAVQ-R)

\author{
PAUL CHADWICK, SUSAN LEES and MAX BIRCHWOOD
}

\begin{abstract}
Background We present a revised Beliefs About Voices Questionnaire (BAVQ-R), a self-report measure of patients' beliefs, emotions and behaviour about auditory hallucinations.
\end{abstract}

\begin{abstract}
Aims To improve measurement of omnipotence, a pivotal concept in understanding auditory hallucinations, and elucidate links between beliefs about voices, anxiety and depression.
\end{abstract}

\begin{abstract}
Methods Seventy-one participants with chronic auditory hallucinations completed the BAVQ-R, and 58 also completed the Hospital Anxiety and Depression Scale.
\end{abstract}

Results The mean Cronbach's $\alpha$ for the five sub-scales was 0.86 (range 0.74-0.88).

The study supports hypotheses about links between beliefs, emotions and behaviour, and presents original data on how these relate to the new omnipotence sub-scale. Original data are also presented on connections with anxiety and depression.

Conclusions The $B A V Q-R$ is more reliable and sensitive to individual differences than the original version, and reliably measures omnipotence.

Declaration of interest This study was supported by a University of Southampton grant to the first author.
Chadwick \& Birchwood (1994) proposed a cognitive model of the maintenance of auditory hallucinations. This model proposed that people's emotional and behavioural reactions to auditory hallucinations reflect not only content and form, but also the meaning given to the auditory hallucinations. Research indicates the importance of beliefs about identity, purpose ('Why me?'), omnipotence and the consequences of compliance or resistance (Chadwick \& Birchwood, 1994; Birchwood \& Chadwick, 1997). The original Beliefs About Voices Questionnaire (BAVQ) was derived from this model and measured beliefs, feelings and behaviour associated with auditory hallucinations. Data from 60 participants showed the measure to be reliable and valid, and offered support for the importance of beliefs (Chadwick \& Birchwood, 1995).

The BAVQ had two specific weaknesses. First, participants answered 'yes' or 'no' to each of the 30 items. For this reason, small individual differences and subtle changes over time were missed. Second, although research shows that people's perception of auditory hallucinations as omnipotent is of central importance, the BAVQ contained but one item specially measuring omnipotence ('My voice is very powerful'). The revised version contains a further five items measuring omnipotence. Here we present data gathered using the new BAVQ-R from a fresh sample of 73 people with chronic, drugresistant auditory hallucinations. These data are linked to scores on the Hospital Anxiety and Depression Scale (HADS) for 58 participants.

\section{METHOD}

\section{Participants}

A fresh sample of 73 people with drugresistant auditory hallucinations participated in the study: 41 men and 32 women, with a mean age of 40 years (s.d. $=10.91)$; all had heard auditory hallucinations for at least 2 years. All had hospital diagnoses of schizophrenia, schizoaffective disorder or psychotic depression. Approximately $60 \%$ of the participants were referred for a psychological assessment for cognitive behaviour therapy, and completed the BAVQ-R as part of this assessment; all the other participants had volunteered to complete the measure and were then asked if they wished to be referred for a psychological assessment of suitability for cognitive behaviour therapy.

\section{Measures}

Beliefs About Voices Questionnaire-Revised

The BAVQ-R is a 35 -item measure of people's beliefs about auditory hallucinations, and their emotional and behavioural reactions to them. There are three sub-scales relating to beliefs: malevolence (six items: e.g. 'My voice is punishing me for something I have done'); benevolence (six items: e.g. 'My voice wants to protect me'); and omnipotence (six items). The five new items assessing omnipotence were obtained over a period of 3 years. The wording of each item reflects statements which are commonly made during psychological assessment or therapy.

Two further sub-scales, 'resistance' and 'engagement', measure emotional and behavioural relationships to auditory hallucinations. 'Resistance' has five items on emotion (e.g. 'My voice frightens me') and four on behaviour (e.g. 'When I hear my voice usually I tell it to leave me alone'). 'Engagement' has four items on emotion (e.g. 'My voice reassures me') and four on behaviour (e.g. 'When I hear my voice usually I listen to it because I want to').

All responses are rated on a 4-point scale: disagree (0); unsure (1); agree slightly (2); agree strongly (3). The measure thus assesses degree of endorsement of items. As with the original BAVQ, individuals hearing more than one auditory hallucination complete the questionnaire for their 'dominant voice'. The BAVQ-R is available from the first author upon request.

\section{Hospital Anxiety and Depression Scale}

The HADS (Zigmond \& Snaith, 1983) is a 14-item self-administered rating scale of depressive (seven items) and anxious (seven items) symptoms. Like all such self-report measures, the HADS is not diagnostic. 
Scores on the HADS for both depressive and anxious symptoms are categorised as follows: normal range, $0-7$; mild, 8-10; moderate, 11-14; severe, 15-21.

\section{Statistical analysis}

For each scale of the BAVQ-R the $\alpha$ coefficient was calculated, thus giving a measure of reliability drawn from a single administration of the measure. We present descriptive statistical analysis of the distribution of scores on all sub-scales (including skewness), and endorsement of each omnipotence item. Pearson correlations are calculated for relationships among the different BAVQ-R sub-scales, and between these and the HADS. All analyses were carried out using SPSS 8 for Windows.

\section{RESULTS}

\section{Descriptive statistics}

In the present study participants used the full range of scores for the sub-scales measuring malevolence, resistance and engagement; no one scored above 16 on the benevolence sub-scale, and no one scored zero on the omnipotence sub-scale. Differences were seen in the distributions of scores, and these differences replicated our previous findings (Chadwick \& Birchwood, 1995). Skewness statistics for the different sub-scales were as follows: malevolence -0.452 , resistance -1.231 , engagement 1.274, benevolence 1.113, and omnipotence -0.180 . Sub-scale means (with standard deviations) and medians were as follows: malevolence, mean 10.1 (s.d.=5.5), median 12; benevolence, mean 3.7 (s.d.=4.7), median 1; omnipotence, mean 11.1 (s.d. $=4.5$ ), median 11; engagement, mean 5 (s.d. $=5.7$ ), median 2; resistance, mean 19.4 (s.d.=6.5), median 21 . (Both means and medians are given, because not all sub-scales showed a normal distribution.)

\section{Reliability and validity of the BAVQ-R}

For each sub-scale of the BAVQ-R the $\alpha$ coefficient was calculated, thus giving a measure of reliability drawn from a single measure. Cronbach's $\alpha$ scores for each sub-scale, including the new omnipotence sub-scale, were uniformly high. Furthermore, comparison with data from our previous study (Chadwick \& Birchwood, 1995) showed that for malevolence,

Table I Psychometric properties of the revised Beliefs About Voices Questionnaire (BAVQ-R) $(n=71)$

\begin{tabular}{|c|c|c|c|c|c|}
\hline & Malevolence & Benevolence & Omnipotence & Resistance & Engagement \\
\hline No. of items & 6 & 6 & 6 & 9 & 8 \\
\hline Range of scores & $0-18$ & $0-18$ & $0-18$ & $0-27$ & $0-24$ \\
\hline $\begin{array}{l}\text { Range of item-item } \\
\text { correlations }\end{array}$ & $0.29-0.69$ & $0.35-0.67$ & $0.07-0.68$ & $0.16-0.75$ & $0.2-0.63$ \\
\hline $\begin{array}{l}\text { Range of item-total } \\
\text { correlations }\end{array}$ & $0.47-0.83$ & $0.55-0.78$ & $0.26-0.6$ & $0.42-0.69$ & $0.5 I-0.74$ \\
\hline Cronbach's $\alpha$ & 0.84 & 0.88 & 0.74 & 0.85 & 0.87 \\
\hline
\end{tabular}

benevolence and resistance, Cronbach's $\alpha$ scores were all higher on the BAVQ-R. For engagement, the Cronbach's $\alpha$ scores were identical. It therefore appears that the BAVQ-R continues to measure clear and stable aspects of individuals' relationships with their auditory hallucinations.

\section{Construct validity}

The correlations between the different subscales of the BAVQ-R were examined. As in previous studies (Chadwick \& Birchwood, 1994, 1995; Birchwood \& Chadwick, 1997), we found a strong relationship between malevolence and resistance $(r=0.68$, d.f. $=69, P<0.01)$ and benevolence and engagement $(r=0.80$, d.f. $=69, P<0.01$ ), with all other correlations between these sub-scales being strongly negative.

\section{Omnipotence scale}

The descriptive statistics that follow take a conservative view of endorsement of an item, as 'Agree slightly' or 'Agree strongly'. The percentage level of endorsement of each item on the omnipotence sub-scale is 'My voice is very powerful' ( $86 \%)$, 'My voice seems to know everything about me' (79\%), 'I cannot control my voice' $(75 \%)$, 'My voice rules my life' (63\%), 'My voice makes me do things I really don't want to do' $(47 \%)$, and 'My voice will harm or kill me if I do disobey or resist it' $(38 \%)$. In relation to the item-total correlations (Table 1), the score for one item ('I cannot control my voices') is a little low (0.26). However, this item is retained in the measure because it is of critical importance clinically, and is one of the most highly endorsed on the entire measure (mean score 2.2).

A two-tailed examination was made of the new omnipotence sub-scale in relation to the other sub-scales of the BAVQ. The relationship between omnipotence and
Table 2 Scores on the Hospital Anxiety and Depression Scale (HADS) (58 participants)

\begin{tabular}{lccc}
\hline & Anxiety & Depression \\
\hline Mean score (s.d.) & $12.97(5)$ & $10.35(4.67)$ \\
Normal range, \% (n) & $16(9)$ & $33(19)$ \\
Mild range, \% (n) & $17(10)$ & $19(11)$ \\
Moderate range, \% (n) & $26(15)$ & $27(16)$ \\
Severe range, \% (n) & $41(24)$ & $21 \quad(12)$ \\
\hline
\end{tabular}

malevolence was found to be strongly positive $(r=0.70$, d.f. $=69, P<0.01)$, as was the relationship between omnipotence and resistance $(r=0.50$, d.f. $=69, P<0.01)$. The sub-scales of omnipotence and engagement had a negative relationship $(r=-0.26$, d.f. $=69, P<0.05)$. There was no significant relationship between the omnipotence and benevolence sub-scales.

\section{Correlations between BAVQ-R and HADS scores}

Fifty-eight participants completed the HADS. Table 2 shows mean scores on the HADS, and the number of participants falling into the different ranges of severity. Previous findings (Chadwick \& Birchwood, 1996) had led us to expect a relationship between malevolence and depressive symptoms (hereafter called simply 'depression'). This was found in the present study $(r=0.37$, d.f. $=56, P<0.01)$. There was also a relationship between depression and both omnipotence $(r=0.44$, d.f. $=56, P<0.01)$ and resistance $(r=0.32$, d.f. $=56, P<0.05)$. Depression was negatively associated with engagement $(r=-0.42$, d.f. $=56, P<0.01)$.

Anxious symptomatology (hereafter called simply 'anxiety') was related to malevolence $(r=0.30$, d.f. $=56, P<0.05)$, resistance $(r=0.40$, d.f. $=56, P<0.01)$ and omnipotence $(r=0.33$, d.f. $=56, P<0.05)$. There was a negative relationship between 
anxiety and engagement $(r=0.36$, d.f. $=56$, $P<0.05)$. Scores on the HADS showed a significant relationship between anxiety and depression $(r=0.59$, d.f. $=56, P<0.01)$.

\section{DISCUSSION}

The present study reports psychometric properties of a revised Beliefs About Voices Questionnaire (BAVQ-R). The revision involved adding five items measuring omnipotence, to create an omnipotence subscale. Also, the original two-point scale was replaced with a four-point scale, to improve detection of individual differences and perhaps increase the usefulness of the scale as a measure of change. Overall, the present study offers a favourable analysis of the BAVQ-R. Cronbach's $\alpha$ scores for all five sub-scales are acceptably high, and either equal to (benevolence) or better (malevolence, resistance and engagement) than those of the original. The present study also replicates the correlations observed between malevolence and resistance, on the one hand, and benevolence and engagement, on the other. These data, combined with those in earlier studies (Birchwood \& Chadwick, 1997; Chadwick \& Birchwood, 1995), suggest a robust analysis of people's relationships with their auditory hallucinations, and again emphasise the central importance of beliefs in shaping these relationships.

\section{Importance of omnipotence}

The central importance of people's perceptions of auditory hallucinations as very powerful was observed first by Bauer (1970). In a seminal paper, he reported how auditory hallucinations can be imbued with a "terrifying and compelling quality" and how individuals can feel "caught in a voice's power" (p. 169). The new omnipotence sub-scale may be thought of as an attempt to operationalise this quality. Descriptive data confirm that omnipotence is a vital part of an analysis of these participants' relationships with their auditory hallucinations.

Omnipotence has the highest mean score and lowest standard deviation (11.1, s.d. $=4.5$ ) of the three sub-scales which measure beliefs. Indeed, so important do we judge omnipotence to be that we have developed and evaluated a group-based cognitive behaviour therapy which principally targets beliefs about omnipotence (Chadwick et al, 2000).

\section{CLINICAL IMPLICATIONS}

Participants continue to find the measure acceptable and easy to complete.

- The BAVQ-R is a useful assessment and outcome measure.

- The measure gives a quick, reliable profile of a person's relationship with an auditory hallucination, which is useful information for psychological therapy.

\section{LIMITATIONS}

- The BAVQ-R assesses relationship with an individual auditory hallucination.

- The BAVQ-R does not assess form or content.

- The cognitive model is of maintenance, not origin, of auditory hallucinations.

PAUL CHADWICK, Head of Clinical Psychology, Royal South Hants Hospital \& Visiting Professor in Clinical Psychology, University of Exeter; SUSAN LEES, Research Assistant, Department of Psychology, University of Southampton; MAX BIRCHWOOD, Director of Early Intervention Service, North Birmingham Mental Health Trust, and Professor of Psychology, University of Birmingham

Correspondence: Paul Chadwick, School of Psychology, University of Exeter, Washington Singer Laboratories, Perry Road, Exeter EX4 4QG, UK

(First received 13 October 1999, final revision 10 February 2000, accepted I5 February 2000)

\section{Defining the concept of omnipotence}

The present study helps to define our concept of omnipotence, which has hitherto been used as loosely equivalent to that of 'power'. Clearly, perceiving an auditory hallucination to be very powerful remains an important attribute of omnipotence. In the present study $86 \%$ of the sample agreed with the statement ' $M y$ voice is very powerful'. Yet $75 \%$ also endorsed the item 'I cannot control my voices', and $79 \%$ the item 'My voices seem to know everything about me'. This makes it clear that the concept of omnipotence implies more than mere power. In future, it may therefore be helpful to refer to power or powerfulness as one specific aspect of omnipotence. The word 'omnipotence' would then be reserved for describing the broader concept, as measured by the BAVQ-R sub-scale.

\section{Does a predominant profile emerge for clinical cases of this sort?}

Data from this and other studies suggest that a 'profile' may be emerging which would describe the majority of patients using psychiatric services who experience auditory hallucinations. First, as in the present study, participants almost invariably perceive their dominant hallucinations as a considerable problem and source of distress, notwithstanding the fact that particular aspects of their relationships with auditory hallucinations may be positive. Second, omnipotence scores are very high (cf. Close \& Garety, 1998). Third, the dominant auditory hallucination is predominantly perceived as malevolent, evoking behavioural resistance and negative affect. Fourth, the person is likely to experience at least a moderate level of anxiety symptoms and mild or moderate depressive symptoms. Yet it is important to note that for many people the experience of auditory hallucinations is a positive one; in a community sample $(n=173)$ of patients and non-patients, Romme \& Escher (1989) found that $15 \%$ of participants perceived their auditory hallucinations as positive.

\section{ACKNOWLEDGEMENTS}

Thanks to the participants, to Professor David Dagnan for statistical advice, and to Drs Bridgette O'Neill, Sarah Brothwell and Sarah Rasch for help with data collection. 


\section{REFERENCES}

Bauer, S. (1970) The function of hallucinations: an enquiry into the relationship of hallucinatory experience to creative thought. In Origin and Mechanisms of Hallucinations (ed.W. Keup). New York: Plenum.

Birchwood, M. J. \& Chadwick, P. D. J. (1997) The omnipotence of voices: testing the validity of a cognitive model. Psychological Medicine, 27, 1345-1353.

Chadwick, P. \& Birchwood, M. (1994) The omnipotence of voices. A cognitive approach to auditor hallucinations. British Journal of Psychiatry, 164, 190-201.
_ \& _ (1995) The omnipotence of voices. II: The Beliefs About Voices Questionnaire. British Journal of Psychiatry, 166, 773-776.

— \& - (1996) Cognitive therapy for voices. In Cognitive-Behavioural Interventions for Psychotic Disorders (eds G. Haddock \& P. D. Slade). London: Routledge.

\section{_, Sambrooke, S., Rasch, S., et al (2000)}

Challenging the omnipotence of voices: group cognitive behaviour therapy for voices. Behaviour Research and Therapy, in press.
Close, H. \& Garety, P. (1998) Cognitive assessments of voices: further developments in understanding the emotional impact of voices. British Journal of Clinical Psychology, 37, 189-197.

Romme, M. \& Escher, S. (1989) Hearing voices. Schizophrenia Bulletin, 15, 209-216.

Zigmond, A. S. \& Snaith, R. P. (1983) The Hospital Anxiety and Depression Scale. Acta Psychiatrica Scandinavica, 67, 36I-370. 